\title{
Utilidad de las escalas de riesgo de sangrado y trombosis en cardiología: ¿cómo y cuándo aplicarlas?
}

\author{
Utility of the bleeding and thrombosis risk scores in cardiology: How and when to apply \\ them?
}

\author{
Gilberto A. Castillo-Barrios ${ }^{1,2 *}$ \\ ${ }^{1}$ Unidad Cardiovascular, Clínica Imbanaco; ${ }^{2}$ Dirección científica, Cardioprevent IPS. Cali, Colombia
}

\section{Introducción}

La terapia antitrombótica es fundamental en el tratamiento de patologías como el síndrome coronario agudo y la fibrilación auricular. Los medicamentos anticoagulantes 0 antiplaquetarios, o la combinación de ellos en casos especiales, son estrategias comunes a las cuales nos enfrentamos en la práctica cardiológica normal.

Sin embargo, el uso de antitrombóticos con el objetivo de reducir las complicaciones isquémicas siempre expone al paciente a un riesgo incrementado de sangrado; es prioritario establecer el beneficio clínico neto de la terapia antitrombótica para definir si el empleo de estos medicamentos ofrece un beneficio antiisquémico superior al riesgo de una hemorragia grave.

Para tal fin, se han diseñado varias escalas que pretenden evaluar el riesgo de trombosis y el riesgo de sangrado para diferentes enfermedades, y que permiten comparar la probabilidad de sufrir una complicación isquémica o hemorrágica, para definir la indicación de la terapia y su duración.

\section{Post-infarto de miocardio}

La revascularización percutánea o quirúrgica, junto con la terapia antiplaquetaria dual, se utilizan para reducir el riesgo de complicaciones isquémicas en los pacientes que han presentado un infarto agudo de miocardio. Sin embargo, esta estrategia también se asocia con un mayor riesgo de sangrado. Además, y parecido a lo que ocurre en la fibrilación auricular, las características clínicas que se asocian con mayor riesgo isquémico, como la edad avanzada y el deterioro de la función renal, también son factores de riesgo para sufrir una hemorragia clínicamente significativa ${ }^{1}$.

A pesar del beneficio antitrombótico, la antiagregación plaquetaria dual se asocia con mayor riesgo de sangrado, lo cual afecta el pronóstico global de los pacientes de una manera similar a las complicaciones isquémicas y trombóticas ${ }^{2}$. Por tal razón, el uso de escalas de riesgo puede ser útil para guiar el tipo y la duración de la antiagregación plaquetaria dual minimizando el riesgo de sangrado.

Las tres escalas de evaluación mas conocidas que pretenden establecer el riesgo isquémico o hemorrágico en los pacientes que han sufrido un evento coronario agudo previo son la DAPT (Dual AntiPlatelet Therapy), la PRECISE-DAPT (PREdicting bleeding Complications In patients undergoing Stent implantation and subsEquent Dual Anti Platelet Therapy) y la ARC-HBR (Academic Research Consortium for High Bleeding Risk). La primera evalúa el riesgo de sufrir una complicación trombótica, mientras que las dos últimas evalúan el riesgo de sangrado.

\section{Correspondencia:}

*Gilberto A. Castillo-Barrios

E-mail: gilberto.castillo@ imbanaco.com.co
Disponible en internet: 10-06-2021 Rev Colomb Cardiol. 2021;28(2):104-106 www.rccardiologia.com 0120-5633 / @ 2021 Sociedad Colombiana de Cardiología y Cirugía Cardiovascular. Publicado por Permanyer. Este es un artículo open access bajo la licencia CC BY-NC-ND (http://creativecommons.org/licenses/by-nc-nd/4.0/). 
La escala DAPT identificó nueve factores asociados con mayor riesgo isquémico cuando se evaluaba después de 12 meses de haber ocurrido el evento previo: edad, disfunción ventricular izquierda o falla cardiaca, stent en injerto venoso, infarto de miocardio como presentación inicial, infarto o angioplastia previa, diabetes mellitus, diámetro del stent $<3 \mathrm{~mm}$, tabaquismo e implante de un stent liberador de paclitaxel. Los pacientes con 2 puntos o más se consideran de alto riesgo isquémico y en ellos hay beneficio de la extensión de la antiagregación dual hasta 30 meses, con un incremento modesto en la tasa de sangrado. En cambio, los pacientes con menos de 2 puntos se consideran de bajo riesgo isquémico y no tienen un beneficio clínico neto positivo que justifique prolongar la antiagregación plaquetaria dual después de los 12 meses $^{3}$.

La escala PRECISE-DAPT identificó cinco factores predictores de alto riesgo de sangrado que se deben evaluar en el momento del alta por el evento agudo: edad, depuración de creatinina, hemoglobina, recuento de leucocitos y sangrado espontáneo. Los pacientes con puntaje de la escala PRECISE-DAPT $\geq 25$ tienen un riesgo alto de sangrado y no se benefician de la terapia antiplaquetaria dual extendida; en cambio, aquellos con puntaje PRECISE-DAPT < 25 tienen una reducción significativa del desenlace cardiovascular combinado con la antiagregación dual prolongada, sin incremento del sangrado 4 .

Finalmente, el ARC-HBR es un documento de consenso que evaluó la eficacia y la seguridad de los medicamentos empleados en los ensayos clínicos que incluyeron pacientes con alto riesgo de sangrado y que fueron llevados a angioplastia coronaria, pero que fueron previamente excluidos de los estudios de antiagregación dual. Sin embargo, la evaluación del riesgo hemorrágico de acuerdo con los criterios del ARC-HBR puede ser difícil de aplicar en la práctica clínica habitual, pues incluye algunos criterios demasiado específicos, como la existencia de una malformación arteriovenosa cerebral, cirrosis hepática con hipertensión portal o diátesis hemorrágica crónica ${ }^{5}$.

Ninguna de estas escalas de predicción del riesgo ha sido evaluada prospectivamente en ensayos clínicos controlados, por lo que aún es incierto su impacto en la mejoría de los desenlaces clínicos de los pacientes. Las guías de la European Society of Cardiology para el manejo de los síndromes coronarios agudos sin elevación del segmento ST recomiendan la doble antiagregación plaquetaria con ácido acetilsalicílico y un inhibidor del receptor P2Y12 (clopidogrel, ticagrelor o prasugrel) por 12 meses después de un evento coronario agudo. No obstante, si el paciente tiene un alto riesgo de sangrado (por ejemplo, un puntaje PRECISE-DAPT $\geq 25$ o criterios de ARC-HBR), podría considerarse la suspensión de la antiagregación dual después de 3 a 6 meses $^{6}$.

\section{Fibrilación auricular}

La fibrilación auricular aumenta hasta cinco veces el riesgo de sufrir un evento cerebrovascular de origen cardioembólico independientemente de otros factores de riesgo, por lo que definir la anticoagulación a largo plazo es una de las prioridades en el manejo de este tipo de pacientes?.

Las guías de fibrilación auricular de la American Heart Association, el American College of Cardiology y la Heart Rhythm Society, así como las guías de la European Society of Cardiology, recomiendan la escala CHA2DS2-VASc para la evaluación del riesgo cardioembólico. Esta escala predice solo modestamente aquellos pacientes de alto riesgo de sufrir un evento cardioembólico, pero logra identificar de manera consistente aquellos con riesgo bajo de muerte y de evento cerebrovascular ${ }^{8}$.

En los últimos años han surgido nuevas escalas de estratificación del riesgo, como ATRIA, ABC y GARDFIELD-AF, que incluyen otros factores de riesgo para sufrir un evento cardioembólico, aunque la mayoría coinciden en incluir la edad avanzada y el haber tenido un evento cerebrovascular previo (o isquemia cerebral transitoria). La escala más recientemente incorporada es la GARDFIELD-AF ${ }^{9}$, la cual permite, mediante una aplicación web, calcular el riesgo de ataque cerebrovascular o embolia sistémica, el riesgo de sangrado mayor y la mortalidad por todas las causas. A pesar de tener una mayor capacidad de predicción, este incremento no es de gran magnitud y tiene la limitación de requerir una herramienta tecnológica para su aplicación, mientras que la mayoría de los médicos calculan el CHA2DS2-VASc de memoria, según una encuesta de la Sociedad Europea del Ritmo Cardiaco publicada en $2020^{10}$.

De modo similar a lo que ocurre con los pacientes que han tenido un evento coronario agudo, los mismos factores que se asocian con mayor riesgo de complicaciones trombóticas están asociados con un incremento en el sangrado: edad avanzada, disfunción renal, hipertensión arterial mal controlada, etc. Al igual que con el riesgo trombótico, también existen varias escalas de evaluación del riesgo hemorrágico en pacientes con fibrilación auricular: HAS-BLED, ATRIA, 
ORBIT y HEMORR2HAGES, entre otras; sin embargo, las guías siguen sugiriendo el empleo de la HASBLED. El propósito de realizar esta evaluación es identificar aquellos pacientes con alto riesgo hemorrágico que pueden tener características modificables o corregibles que, al ser intervenidas, minimicen el riesgo de sangrado.

Igualmente, es importante recordar que el riesgo de complicaciones isquémicas y hemorrágicas cambia con el tiempo en la medida en que el paciente se hace mayor y aparece nueva comorbilidad, por lo que es importante una reevaluación periódica del riesgo para garantizar los mejores resultados clínicos para los pacientes.

\section{Conflictos de intereses}

El autor declara que no existen conflictos de intereses.

\section{Bibliografía}

1. Mukherjee D. Tailoring strategies to optimize ischaemic benefit vs. bleeding risk in individuals post-myocardial infarction. Eur Heart J. 2020;41:844-6
2. Ducrocq G, Schulte PJ, Budaj A, Cornel JH, Held C, Himmelmann A et al. Balancing the risk of spontaneous ischemic and major bleeding events in acute coronary syndromes. Am Heart J. 2017;186:91-9.

3. Yeh RW, Secemsky EA, Kereiakes DJ, Normand SL, Gershlick AH, Cohen DJ, et al. Development and validation of a prediction rule for benefit and harm of dual antiplatelet therapy beyond 1 year after percutaneous coronary intervention. JAMA. 2016;315:1735-49.

4. Costa F, van Klaveren D, James S, Heg D, Räber L, Feres F, et al. Derivation and validation of the predicting bleeding complications in patients undergoing stent implantation and subsequent dual antiplatelet therapy (PRECISE-DAPT) score: a pooled analysis of individual-patient datasets from clinical trials. Lancet. 2017;389:1025-34.

5. Urban P, Mehran R, Colleran R, Angiolillo DJ, Byrne RA, Capodanno D, et al. Defining high bleeding risk in patients undergoing percutaneous coronary intervention: a consensus document from the Academic Research Consortium for High Bleeding Risk. Eur Heart J. 2019;40:2632-53.

6. Collet JP, Thiele H, Barbato E, Barthélémy O, Bauersachs J, Bhatt DL, et al. 2020 ESC Guidelines for the management of acute coronary syndromes in patients presenting without persistent ST-segment elevation. Eur Heart J. 2020 Aug 29:ehaa575. doi: 10.1093/eurheartj/ehaa575. Online ahead of print.

7. Lane DA, Lip GYH. Stroke and bleeding risk stratification in atrial fibrillation: a critical appraisal. Eur Heart J Suppl. 2020;22(Suppl O):014-27.

8. Hindricks G, Potpara T, Dagres N, Arbelo E, Bax JJ, Blomström-Lundqvist C, et al. 2020 ESC Guidelines for the diagnosis and management of atrial fibrillation developed in collaboration with the European Association for Cardio-Thoracic Surgery (EACTS). Eur Heart J. 2021;42:373-498.

9. Fox KAA, Lucas JE, Pieper KS, Bassand JP, Camm AJ, Fitzmaurice DA, et al. Improved risk stratification of patients with atrial fibrillation: an integrated GARFIELD-AF tool for the prediction of mortality, stroke and bleed in patients with and without anticoagulation. BMJ Open. 2017;7:e017157.

10. Zhang J, Lenarczyk R, Marin F, Malaczynska-Rajpold K, Kosiuk J, Doehner W, et al. Interpretation of the CHA2DS2-VASc score components in clinical practice: a joint survey by the European Heart Rhythm Association (EHRA) Scientific Initiatives Committee, the EHRA Young Electrophysiologists, the Association of Cardiovascular Nursing and Allied Professionals, and the European Society of Cardiology Council on Stroke. Europace. 2021;23:314-22. 